\title{
ПРОЦЕДУРА ИДЕНТИФИКАЦИИ ДЛЯ ЛИНЕЙНЫХ ДИСКРЕТНЫХ СТАЦИОНАРНЫХ СИСТЕМ ПО ДАННЫМ вХОД-вЫХОД
}

\section{1. Постановка задачи}

Рассматривается дискретная стационарная линейная система с одним входом и одним выходом, описываемая разностным уравнением

$$
y(t)=\sum_{i=1}^{n} a_{i} y(t-i)+\sum_{i=1}^{n} b_{i} u(t-i),
$$

где $y(t)$ и $u(t)$ - выход и вход системы. Предполагается, что входы и выходы измеряются с ошибками

$$
\tilde{y}(t)=y(t)+\varepsilon_{y}(t), \quad \tilde{u}(t)=u(t)+\varepsilon_{u}(t),
$$

причем

$$
\begin{array}{ll}
E \varepsilon_{y}(t)=E \varepsilon_{u}(t)=0, & E \varepsilon_{y}(t) \varepsilon_{y}(s)=\delta_{t s} \sigma_{y}^{2}, \\
E \varepsilon_{u}(t) \varepsilon_{u}(s)=\delta_{t s} \sigma_{u}^{2}, & E \varepsilon_{y}(t) \varepsilon_{u}(s)=0,
\end{array}
$$

где $\sigma_{y}$ и $\sigma_{u}$ известные величины.

Требуется определить порядок системы $n$ и оценить неизвестные параметры $a_{1}, a_{2}, \ldots, a_{n}, b_{1}, b_{2}, \ldots, b_{n}$, используя наблюдения над последовательностями $\tilde{y}(t)$ и $\tilde{u}(t)$ при $t=1,2, \ldots, K$, где $K \gg 1$.

\section{2. Обсуждение}

Задача оценки параметров системы (1) ранее $\left[{ }^{1-5}\right]$ рассматривалась в предположении, что ее порядок известен заранее. В настоящей работе порядок системы определяется на основе наблюдаемых данных входа и выхода. Предлагаемый метод в комбинашии с методом собственного вектора оценки неизвестных параметров $\left[{ }^{2-4}\right]$ позволяет идентифицировать порядок и параметры системы одновременно, т. е. порядок определять через наименьшее собственное значение некоторой матрицы, а оценки параметров получать как компоненты собственного вектора, соответствующего этому наименьшему собственному значению. Отметим, что вычисление указанного собственного значения дает одновременно и отвечающий ему собственный вектор. Метод определения порядка, использующий статистические свойства ошибок измерения, основан на теории возмущений однократных собственных значений.

Из многих методов оценки параметров метод собственного вектора выбран из тех соображений, что при сравнительной простоте он дает обычно удовлетворительные результаты $[2,4]$. 


\section{3. Идентификация системы}

В дальнейшем будут использованы следующие обозначения:

$$
\begin{aligned}
& \mathrm{s}_{l i}=[y(i+l) y(i+l-1) \ldots y(i) u(i+l-1) u(i+l-2) \ldots u(i)]^{\prime}, \\
& \mathrm{x}_{l i}=[\widetilde{y}(i+-l) \tilde{y}(i+l-1) \ldots \tilde{y}(i) \tilde{u}(i+l-1) \tilde{u}(i+l-2) \ldots \tilde{u}(i)]^{\prime} \text {, } \\
& \varepsilon_{i}=\left[\varepsilon_{y}(i+n) \varepsilon_{y}(i+n-1) \ldots \varepsilon_{y}(i) \varepsilon_{u}(i+n-1) \varepsilon_{u}(i+n-2) \ldots \varepsilon_{u}(i)\right]^{\prime} \text {, } \\
& \Theta=\left[\begin{array}{lllllllll}
-1 & a_{1} & a_{2} & \ldots & a_{n} & b_{1} & b_{2} & \ldots & b_{n}
\end{array}\right]^{\prime},
\end{aligned}
$$

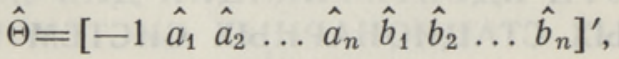

$\mathrm{I}_{l}-$ единичная матрица порядка $l$,

$$
\begin{aligned}
& \mathrm{C}_{l}=\left[\begin{array}{c:c}
\frac{1}{\sigma_{y}} \mathrm{I}_{l+1} & 0 \\
0 & \frac{1}{\sigma_{u}} \mathrm{I}_{l}
\end{array}\right], \\
& \Phi_{l}=\left[\begin{array}{c}
s^{\prime}{ }_{l 1} \\
s_{l 2}^{\prime} \\
\cdot \\
s_{l N}^{\prime}
\end{array}\right], \quad \tilde{\Phi}_{l}=\left[\begin{array}{c}
\mathrm{x}^{\prime}{ }_{l} \\
\mathrm{x}_{l 2}^{\prime} \\
\cdot \\
\cdot \\
\mathrm{x}_{l N}^{\prime}
\end{array}\right], \quad \mathrm{V}_{n}=\left[\begin{array}{c}
\varepsilon^{\prime}{ }_{1} \\
\varepsilon_{2}^{\prime} \\
\cdot \\
\cdot \\
\varepsilon^{\prime}{ }_{N}
\end{array}\right] \text {, } \\
& \mathrm{A}=\frac{1}{N} \mathrm{C}_{n} \Phi^{\prime}{ }_{n} \Phi_{n} \mathrm{C}_{n}+\mathrm{I}_{2 n+1}, \\
& \mathrm{~B}=\frac{1}{N}\left(\mathrm{C}_{n} \Phi^{\prime}{ }_{n} \mathrm{~V}_{n} \mathrm{C}_{n}+\mathrm{C}_{n} \mathrm{~V}^{\prime}{ }_{n} \Phi_{n} \mathrm{C}_{n}\right) \text {, } \\
& \mathrm{D}=\frac{1}{N} \mathrm{C}_{n} \mathrm{~V}_{n}^{\prime} \mathrm{V}_{n} \mathrm{C}_{n}-\mathrm{I}_{2 n+1} \text {. }
\end{aligned}
$$

Оценивание неизвестных параметров. Метод собственного вектора оценки неизвестных параметров состоит в поиске минимума величины

$$
1 / N \sum_{i=1}^{N}\left(\mathrm{x}_{n i}-\mathrm{s}_{n i}\right)^{\prime} \mathrm{C}_{n}^{21}\left(\mathrm{x}_{n i}-\mathrm{s}_{n i}\right)
$$

по векторам $\mathrm{s}_{n i}$ при ограничениях

$$
\Theta^{\prime} \mathrm{s}_{n i}=0, \quad i=1,2, \ldots, N,
$$

т. е. использует принцип взвешанных наименьших квадратов, часто применяемый в статистике. Такая условная минимизация эквивалентна минимизации величины

$$
\frac{1}{N} \sum_{i=1}^{N} \frac{\Theta^{\prime} x_{n i} x^{\prime}{ }_{n i} \Theta}{\Theta^{\prime} C_{n}^{-2} \Theta}=\frac{\Theta^{\prime}\left(\frac{1}{N} \tilde{\Phi}_{n}^{\prime} \tilde{\Phi}_{n}\right) \Theta}{\Theta^{\prime} C_{n}^{-2} \Theta}
$$

по вектору $\Theta$. Последняя задача является обобщенной проблемой собственных значений, так как вектор $\hat{\Theta}$, минимизирующий величину (3), есть решение системы линейных уравнений 


$$
\left(\frac{1}{N} \tilde{\Phi}_{n}^{\prime} \tilde{\Phi}_{n}-\lambda_{\min } \mathrm{C}_{n}^{-2}\right) \hat{\Theta}=0,
$$

где $\lambda_{\min }-$ минимальное решение уравнения

$$
\operatorname{det}\left(\frac{1}{N} \tilde{\Phi}_{n}^{\prime} \tilde{\Phi}_{n}-\lambda \mathrm{C}_{n}^{-2}\right)=0 .
$$

Определение порядк а си стемы. Воспользуемся тем, что матрицы $\Phi_{l}^{\prime} \Phi_{l}$ при $l=1,2, \ldots, n-1$ невырожденны, а матрица $\Phi_{n}^{\prime} \Phi_{n}$ порядка $(2 n+1) \times(2 n+1)$ имеет единственное нулевое собственное значение, если только столбцы входов линейно независимы $\left[{ }^{6}\right]$. Однако нам доступны лишь матрицы $\tilde{\Phi}_{l}^{\prime} \tilde{\Phi}_{l}$. Так как умножение на невырожденную матрицу $C_{l}$ не меняет ее ранга, то вместо матрицы $\tilde{\Phi}_{l}^{\prime} \tilde{\Phi}_{l}$ рассмотрим матрицу $\frac{1}{N} \mathrm{C}_{l} \tilde{\Phi}_{l}^{\prime} \tilde{\Phi}_{l} \mathrm{C}_{l}$. Введение матрицы $\mathrm{C}_{l}$ и коэффициента $1 / N$ позволяет определять порядок и оценки параметров в процессе единой процедуры, поскольку собственные значения матрицы $\frac{1}{N} \mathrm{C}_{n} \tilde{\Phi}_{n}^{\prime} \tilde{\Phi}_{n} \mathrm{C}_{n}$ равны корням уравнения

$$
\operatorname{det}\left(\frac{1}{N} \tilde{\Phi}_{n}^{\prime} \tilde{\Phi}_{n}-\lambda C_{n}^{-2}\right)=0
$$

Ноль не является собственным значением матрицы $\frac{1}{N} \mathrm{C}_{n} \tilde{\Phi}_{n}^{\prime} \tilde{\Phi}_{n} \mathrm{C}_{n}$, но среди ее собственных чисел есть одно значительно меньше остальных. В случае знания распределения этого собственного значения $\lambda_{\min }$ мы могли бы указать границу $p_{n}$ такую, что вероятность $P_{n}=$ $=P\left\{\lambda_{\min } \leqslant p_{n}\right\}$ была бы достаточно большой. Затем мы могли бы просмотреть последовательность минимальных собственных значений $\lambda_{2 l+1}$ матриц $\frac{1}{N} \mathrm{C}_{l} \tilde{\Phi}_{l}^{\prime} \tilde{\Phi}_{l} \mathrm{C}_{l}$ при $l=1,2, \ldots$ и принять минимальное собственное значение этой матрицы равным нулю, если $\lambda_{2 l+1}<p_{l}$.

Распределение нам, однако, неизвестно и поэтому верхнюю границу построим с помощью теории возмущений. Прежде всего заметим, что

$$
\frac{1}{N} \mathrm{C}_{n} \tilde{\Phi}_{n}^{\prime} \tilde{\Phi}_{n} \mathrm{C}_{n}=\mathrm{A}+\mathrm{B}+\mathrm{D} .
$$

B работе $\left[{ }^{2}\right]$ доказано, что матрицы B и D приближаются к нулю почти наверное при $N \rightarrow \infty$. Следовательно, при достаточно больших $N$ мы можем рассматривать матрицу B +D как возмущение матрицы A.

В Приложении приведены математические ожидания и дисперсии матриц В и D. Показано, что элементы В есть величины порядка $\sqrt{\alpha / N}$, а элементы $\mathrm{D}-$ порядка $\sqrt{1 / N}$, где $\alpha$ - максимальный элемент матрицы А.

Введем нормирующую константу $\mu$ такую, что $\mu^{-1}$ есть максимальный из элементов матриц $\mathrm{A}, \sqrt{\alpha N \mathrm{~B}}, \alpha \sqrt{N} \mathrm{D}$ и $\mathrm{A}+\mathrm{B}$. Это позволит сделать элементы этих матриц абсолютно меньше единицы и, следовательно, позволит использовать формулу для возмущенного собственного значения.

Рассматривая матрицу $\mu \mathrm{B}$ как возмущение матрицы $\mu \mathrm{A}$ и матрицу $\mu \mathrm{D}$ как возмущение матрицы $\mu(\mathrm{A}+\mathrm{B})$, имеем $\left[{ }^{7}\right]$ 


$$
\mu \lambda_{\min }=\mu+\sqrt{1 / \alpha N} k_{1}+O(1 / \alpha \sqrt{N)},
$$

где $k_{1}=\frac{\mathrm{x}^{\prime} \mu \sqrt{\alpha N} \mathrm{Bx}}{\mathrm{x}^{\prime} \mathrm{x}} \quad$ и $\mathrm{x}-$ собственный вектор матрицы $\mathrm{A}$, соответствующий ее минимальному собственному значению, т. е. единице. Из условий (2) получим

$$
\Phi_{n} \mathrm{C}_{n} \mathrm{C}_{n}^{-1} \Theta=0
$$

откуда

$$
\frac{1}{N} \mathrm{C}_{n} \Phi_{n}^{\prime} \Phi_{n} \mathrm{C}_{n} \mathrm{C}_{n}^{-1} \Theta+\mathrm{C}_{n}^{-1} \Theta=\mathrm{C}_{n}^{-1} \Theta \quad \text { и } \quad \mathrm{AC}_{n}^{-1} \Theta=\mathrm{C}_{n}^{-1} \Theta
$$

Следовательно, $\mathrm{x}=\mathrm{C}_{n}^{-1} \Theta$. С учетом формулы (4), $k_{1}=0$ и так как $\mu=O\left(\alpha^{-1}\right)$, можем записать

$$
\lambda_{\min }=1+O(1 / \sqrt{N}) .
$$

Остается определить подходящую константу $\gamma_{l}$ для придания точного значения величине $O(1 / \sqrt{N})$. Заметим, что это весьма тонкая задача. Если возьмем $\gamma_{l}$ слишком малой, есть опасность получить завышенный порядок системы, так как вероятность того, что $\lambda_{\min }>1+\gamma_{l} / \sqrt{N}$, велика. Наоборот, если выберем $\gamma_{l}$ слишком большой, можно получить заниженный порядок системы, особенно в тех случаях, когда некоторые из минимальных собственных значений матриц $\frac{1}{N} \mathrm{C}_{l} \Phi^{\prime}{ }_{l} \Phi_{l} \mathrm{C}_{l}+\mathrm{I}_{2 l+1}$ при $l=1,2, \ldots, n-1$ приблизительно равны единице. Но при увеличении $N$ вероятность такой ошибки уменьшается.

Предварительный опыт моделирования на ЭВМ процедуры идентификации порядка разных систем показал, что $\gamma_{l}$ целесообразно выбирать $\approx 4 \sqrt{l}$.

\section{Приложение}

Обозначим элементы матрицы $\frac{1}{N} \mathrm{C}_{n} \mathrm{~V}_{n}^{\prime} \mathrm{V}_{n} \mathrm{C}_{n}$ через $v_{l r}$.

\section{Поскольку}

$$
v_{l r}= \begin{cases}\frac{1}{N \sigma_{y}^{2}} \sum_{i=1}^{N} \varepsilon_{y}(i+n+1-l) \varepsilon_{y}(i+n+1-r), & l \leqslant n+1, r \leqslant n+1, \\ \frac{1}{N \sigma_{y} \sigma_{u}} \sum_{i=1}^{N} \varepsilon_{u}(i+2 n+1-l) \varepsilon_{y}(i+n+1-r), \quad l>n+1, \quad r \leqslant n+1, \\ \frac{1}{N \sigma_{y} \sigma_{u}} \sum_{i=1}^{N} \varepsilon_{y}(i+n+1-l) \varepsilon_{u}(i+2 n+1-r), l \leqslant n+1, r>n+1, \\ \frac{1}{N \sigma_{u}^{2}} \sum_{i=1}^{N} \varepsilon_{u}(i+2 n+1-l) \varepsilon_{u}(i+2 n+1-r), l>n+1, r>n+1,\end{cases}
$$

то при имеющихся предположениях об ошибках $\varepsilon_{y}(t)$ и $\varepsilon_{u}(t)$ 


$$
E v_{l r}= \begin{cases}1, & l=r \\ 0, & l \neq r\end{cases}
$$

Дисперсия имеет вид

$$
D v_{l r}=E v_{l r}^{2}-\left(E v_{l r}\right)^{2} .
$$

Так как

$$
v_{l r}^{2}=\left\{\begin{array}{l}
\frac{1}{N^{2} \sigma_{y}^{4}} \sum_{i, j=1}^{N} \varepsilon_{y}(i+n+1-l) \varepsilon_{y}(i+n+1-r) \times \\
\times \varepsilon_{y}(j+n+1-l) \varepsilon_{y}(j+n+1-r), \\
\frac{1}{N^{2} \sigma_{y}^{2} \sigma_{u}^{2}} \sum_{i, j=1}^{N} \varepsilon_{u}(i+2 n+1-l) \varepsilon_{y}(i+n+1-r) \times \\
\times \varepsilon_{u}(j+2 n+1-l) \varepsilon_{y}(j+n+1-r), \quad r \leqslant n+1, \\
\frac{1}{N^{2} \sigma_{y}^{2} \sigma_{u}^{2}} \sum_{i, j=1}^{N} \varepsilon_{y}(i+n+1-l) \varepsilon_{u}(i+2 n+1-r) \times \\
\times \varepsilon_{y}(j+n+1-l) \varepsilon_{u}(j+2 n+1-r), \\
\frac{1}{N^{2} \sigma_{u}^{4}} \sum_{i, j=1}^{N} \varepsilon_{u}(i+2 n+1-l) \varepsilon_{u}(i+2 n+1, \quad r>n+1, \\
\times \varepsilon_{u}(j+2 n+1-l) \varepsilon_{u}(j+2 n+1-r), \\
l>n+1, \quad r>n+1,
\end{array}\right.
$$

то

$$
D v_{l r}=\left\{\begin{array}{l}
\frac{1}{N}\left[\frac{E \frac{1}{N} \sum_{i=1}^{N} \varepsilon_{y}^{4}(i+n+1-l)}{\sigma_{y}^{4}}-1\right], \quad l=r \leqslant n+1, \\
\frac{1}{N}\left[\frac{E \frac{1}{N} \sum_{i=1}^{N} \varepsilon_{u}^{4}(i+2 n+1-l)}{\sigma_{u}^{4}}-1\right], \quad l=r>n+1, \\
\frac{1}{N}, l \neq r .
\end{array}\right.
$$

Когда ошибки распределены нормально, то

$$
\begin{aligned}
& \frac{1}{N}\left[\frac{E \frac{1}{N} \sum_{i=1}^{N} \varepsilon_{y}^{4}(i+n+1-l)}{\sigma_{y}^{4}}-1\right]= \\
= & \frac{1}{N}\left[\frac{E \frac{1}{N} \sum_{i=1}^{N} \varepsilon_{u}^{4}(i+2 n+1-l)}{\sigma_{u}^{4}}-1\right]=\frac{2}{N} .
\end{aligned}
$$


Обозначим элементы матрицы $\frac{1}{N} \mathrm{C}_{n} \Phi_{n}^{\prime} \mathrm{V}_{n} \mathrm{C}_{n}$ через ${ }_{1} w_{l r}$ и элементы матрицы $\frac{1}{N} \mathrm{C}_{n} \mathrm{~V}_{n}^{\prime} \Phi_{n} \mathrm{C}_{n}$ через ${ }_{2} w_{l r}$. Тогда

$$
\begin{aligned}
& E_{1} w_{l r}= E_{2} w_{l r}=0, \\
& D_{1} w_{l r}= \begin{cases}\frac{1}{N}\left[\frac{1}{N \sigma_{y}^{2}} \sum_{i=1}^{N} y^{2}(i+n+1-l)\right], & l \leqslant n+1, \\
\frac{1}{N}\left[\frac{1}{N \sigma_{u}^{2}} \sum_{i=1}^{N} u^{2}(i+2 n+1-l)\right], & l>n+1,\end{cases} \\
& D_{2} w_{l r}= \begin{cases}\frac{1}{N}\left[\frac{1}{N \sigma_{y}^{2}} \sum_{i=1}^{N} y^{2}(i+n+1-r)\right], & r \leqslant n+1, \\
\frac{1}{N}\left[\frac{1}{N \sigma_{u}^{2}} \sum_{i=1}^{N} u^{2}(i+2 n+1-r)\right], & r>n+1 .\end{cases} \\
& E_{1} w_{l r} w_{l r}= \begin{cases}\frac{1}{N}\left[\frac{1}{N \sigma_{y}^{2}} \sum_{i=1}^{N} y(i+n+1-l) y(i+n+1-2 r+l)\right], \\
\frac{1}{N}\left[\frac{1}{N \sigma_{u}^{2}} \sum_{i=1}^{N} u(i+2 n+1-l) u(i+2 n+1-2 r+l)\right], \\
0 \quad \text { в остальных случаях. } \quad l>n+1, \quad r>n+1,\end{cases}
\end{aligned}
$$

В последней формуле считаем $y(s)=0$ при $s>N+n, s<0$ и $u(s)=0$ при $s>N+n-1$ или $s<0$.

\section{Л И Т Е Р А Т У Р А}

1. A o k i, M., Y u e, P. C., SIAM J. Control, 8, No. 2, 239 (1970).

2. A o k i, M., Yue, P. C., IEEE Trans. on Autom. Control, AC-15, No. 5, 541 (1970).

3. L evi n, M. J., IEEE Trans. on Autom. Control, AC-9, No. 3, 229 (1964).

4. S mith, F. W.,Hilto n, W. B., IEEE Trans. on Autom. Control, AC-12, No. 5, 568 (1967)

5. Steiglitz, K., McBride, L. E., IEEE Trans. on Autom. Control, AC-10, No. 4, 461 (1965).

6. B u d i n, M. A., IEEE Trans, on Autom. Control, AC-16, No. 5, 395 (1971).

7. У и лкинсон Дж., Алгебраическая проблема собственных значений, М., 1970.

Ннститут кибернетики Академии наук Эстонской ССР
Поступила в редакцию 5/VIII 1976 
olle KOTTA

\section{LINEAARSE STATSIONAARSE DISKREETSE SUSTEEMI IDENTIFITSEERIMINE SISEND-VÄLJUNDANDMETE PŌHJAL}

Vaadeldakse lineaarse statsionaarse diskreetse süsteemi identifitseerimist ja esitatakse meetod sellise süsteemi järgu määramiseks veaga mõõdetud sisend- ja väljundsignaalide alusel. Järk määratakse sisend- ja väljundandmetest moodustatud maatriksi minimaalse omaväärtuse kaudu. Selle meetodi peamine väärtus on selles, et ta moodustab ühe parameetrite hindamise meetodiga - omavektorimeetodiga - ühtse protseduuri, sest süsteemi parameetrite hinnangute vektor on eespool mainitud minimaalsele omaväärtusele vastav omavektor.

\section{ULLE KOTTA}

\section{A PROCEDURE FOR IDENTIFICATION OF LINEAR STATIONARY DISCRETE TIME SYSTEM FROM INPUT-OUTPUT DATA}

The problem of the identification of a linear stationary discrete time system is examined. A method is presented for determining the order of such a system from the noisy measurements of the input and output signals by the least eigenvalue of a certain matrix formed from the input and output signals. The main feature of the method is that it forms a unique procedure with eigenvector method for system parameter estimation as the parameters of the system are estimated by the eigenvector corresponding to the least eigenvalue of considered matrix. 